\title{
Solution solide d'apatites de plomb, d'argent et de sodium : synthèse, caractérisation structurale et propriétés électriques
}

\author{
M. Mehnaoui ${ }^{1}$, R.Ternane ${ }^{1}$, A. Madani ${ }^{2}$ et M. Trabelsi-Ayadi ${ }^{1}$ \\ ${ }^{1}$ Laboratoire de Physico-Chimie Minérale, Faculté des Sciences de Bizerte, 7021 Zarzouna \\ Bizerte, Tunisie \\ ${ }^{2}$ Laboratoire de Physique des Matériaux, Faculté des Sciences de Bizerte, 7021 Zarzouna \\ Bizerte, Tunisie
}

\begin{abstract}
A continuous solid solution of $\mathrm{Pb}_{8} \mathrm{Na}_{2-\mathrm{x}} \mathrm{Ag}_{\mathrm{x}}\left(\mathrm{PO}_{4}\right)_{6}(0 \leq \mathrm{x} \leq 2)$ crystallizing in the anionicdeficient apatite structure was obtained by solid-state reaction. The samples were characterized by using $\mathrm{X}$-ray diffraction, infrared absorption spectroscopy, Raman scattering spectroscopy, ${ }^{31} \mathrm{P}$ MAS-NMR and chemical analysis. The electrical conductivity versus $\mathrm{x}$ measured in a wide range of temperature increases with silver content. For each composition, conductivity values obey the Arrhenius law. Correlations between electrical and structural properties are established.
\end{abstract}

\section{INTRODUCTION}

Le domaine de la recherche propre au transport ionique dans les électrolytes solides a connu une évolution très rapide grâce à la découverte de nombreux composés conducteurs ioniques tels que les superconducteurs ioniques dont la conductivité est suffisamment élevée pour envisager des applications réelles comme électrolytes solides dans les batteries. L'étude des matériaux à faible conductivité ionique reste néanmoins intéressante pour élucider certains mécanismes de transport.

Les apatites forment une grande famille de composés solides qui cristallisent, généralement, dans le système hexagonal de groupe d'espace $\mathrm{P}_{3} / \mathrm{m}$ [1]. Ces apatites ont la formule générale $\mathrm{M}_{10}\left(\mathrm{XO}_{4}\right)_{6} \mathrm{Y}_{2}$ où $\mathrm{M}$ désigne un cation bivalent se répartissant en deux sites différents : $\mathrm{M}(\mathrm{I})$ et $\mathrm{M}(\mathrm{II}),\left(\mathrm{XO}_{4}\right)$ un anion le plus souvent trivalent et $\mathrm{Y}$ un anion monovalent. Une des caractéristiques essentielles de cette structure est sa capacité à former des solutions solides et à accepter un grand nombre de substituants. Les domaines d'utilisation possible du minéral «apatite » sont nombreux et divers :

- la médecine : matériau de comblement osseux, revêtement d'implants dentaires.

- l'agrochimie: engrais chimiques.

- la technologie : -lampes fluorescentes et matériaux laser. -catalyseurs et détecteurs de gaz, en raison de l'existence de la conduction ionique.

- l'industrie nucléaire : matrice de confinement des éléments radioactifs et des produits de fission.

Seules les apatites au plomb sont connues pour être lacunaires en anions $\mathrm{Y}$, il apparaît que l'ion $\mathrm{Pb}^{2+}$ joue un rôle essentiel permettant de conserver le réseau apatitique idéal. Ce rôle est lié à la présence 
des doublets électroniques $6 s^{2}$ qui peuvent compenser le déséquilibre coulombien dû à l'existence des lacunes anioniques dans les tunnels des apatites [2-5].

Les propriétés électriques des apatites $\mathrm{M}_{10}\left(\mathrm{PO}_{4}\right)_{6} \mathrm{Y}_{2}$ ont été largement étudiées. Il a été considéré que les fluoroapatites $\mathrm{Pb}_{10}\left(\mathrm{PO}_{4}\right)_{6} \mathrm{~F}_{2}$ et les hydroxyapatites $\mathrm{Pb}_{10}\left(\mathrm{PO}_{4}\right)_{6}(\mathrm{OH})_{2}$ de plomb sont meilleures conductrices que leurs correspondantes calciques et barytiques. De plus, pour le cas particulier des apatites phosphoplombeuses, il a été montré que la fluoroapatite présente des propriétés de conduction plus performantes que celles de l'hydroxyapatite correspondante [6]. Ce travail est une contribution à une meilleure connaissance des apatites phosphoplombeuses mixtes d'argent et de sodium sans anions $\mathrm{Pb}_{8} \mathrm{Na}_{2-\mathrm{x}} \mathrm{Ag}_{\mathrm{x}}\left(\mathrm{PO}_{4}\right)_{6}(0 \leq \mathrm{x} \leq 2)$ et a également pour objet l'étude de leurs propriétés électriques pour mieux élucider les paramètres gérant la conduction purement cationique des apatites. La mesure de la conductivité ionique pour les différentes compositions est réalisée par la spectroscopie d'impédance complexe.

\section{CONDITIONS EXPÉRIMENTALES}

Dans l'objectif de préparer une solution solide d'apatites phosphoplombeuses mixtes d'argent et de sodium, la réaction à l'état solide et à haute température a été utilisée [4].

Le schéma réactionnel est décrit comme suit :

$$
\begin{aligned}
& 8 \mathrm{PbO}+(2-\mathrm{x}) / 2 \mathrm{Na}_{2} \mathrm{CO}_{3}+\mathrm{x} / 2 \mathrm{Ag}_{2} \mathrm{CO}_{3}+6\left(\mathrm{NH}_{4}\right)_{2} \mathrm{HPO}_{4} \rightarrow \mathrm{Pb}_{8} \mathrm{Na}_{2-\mathrm{x}} \mathrm{Ag}_{\mathrm{x}}\left(\mathrm{PO}_{4}\right)_{6}+\mathrm{CO}_{2}+12 \mathrm{NH}_{3}+ \\
& 9 \mathrm{H}_{2} \mathrm{O}
\end{aligned}
$$

Le dosage des ions phosphate a été réalisé par une méthode décrite par Gee et al. [7] qui consiste en une complexation de ces derniers en un complexe phosphovanadomolybdique suivie par des mesures colorimétriques. Les ions $\mathrm{Pb}^{2+}, \mathrm{Ag}^{+}, \mathrm{Na}^{+}$ont été dosés par spectrométrie d'absorption atomique, à l'aide d'un spectromètre Perkin-Elmer 560.

Les diagrammes des rayons $\mathrm{X}$ de poudre ont été réalisés sur un diffractomètre $\mathrm{D} 8$ advance fonctionnant à la longueur d'onde $\mathrm{K}_{\alpha}$ du cuivre $(\lambda=1,5406 \AA)$. Les spectres d'absorption infrarouge ont été obtenus en utilisant un spectromètre de marque Perkin-Elmer FT-IR Spectrum 1000. L'appareil utilisé pour l'enregistrement des spectres de diffusion Raman est un triple monochromateur de marque Jobin-Yvon. Les mesures de la conductivité ionique ont été effectuées sur des échantillons sous forme de pastilles de diamètre $13 \mathrm{~mm}$ obtenues à partir de poudres cristallisées densifiées sous une pression de 3 à 4 tonnes. $\mathrm{cm}^{-2}$, ensuite frittées à une température de $973 \mathrm{~K}$ pendant 12 heures. L'appareil utilisé est un analyseur d'impédance de type Hewlett-Packard 4192A dans une gamme de fréquences comprises entre $5 \mathrm{~Hz}$ à $13 \mathrm{MHz}$.

\section{RÉSULTATS ET DISCUSSION}

\subsection{Caractérisation physico-chimique}

L'analyse chimique de l'ensemble des composés préparés, montre que les apatites obtenues sont sensiblement stoechiométriques.

Les diffractogrammes des rayons $\mathrm{X}$ présentent des raies fines indexables dans le système hexagonal $\mathrm{P}_{3} / \mathrm{m}$ propres aux apatites. L'augmentation progressive des paramètres cristallographiques et par conséquent du volume de la maille (Figure 1) à mesure que l'apatite s'enrichit en argent

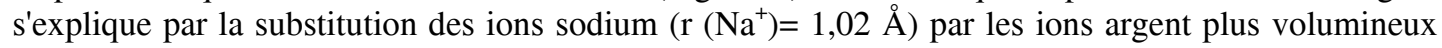


$\left(\mathrm{r}\left(\mathrm{Ag}^{+}\right)=1,28 \AA\right)$. En outre, la variation linéaire de ces grandeurs permet de conclure que ces dernières suivent la loi de Végard et que les échantillons préparés forment bien une solution solide continue d'apatites phosphoplombeuses d'argent et de sodium.
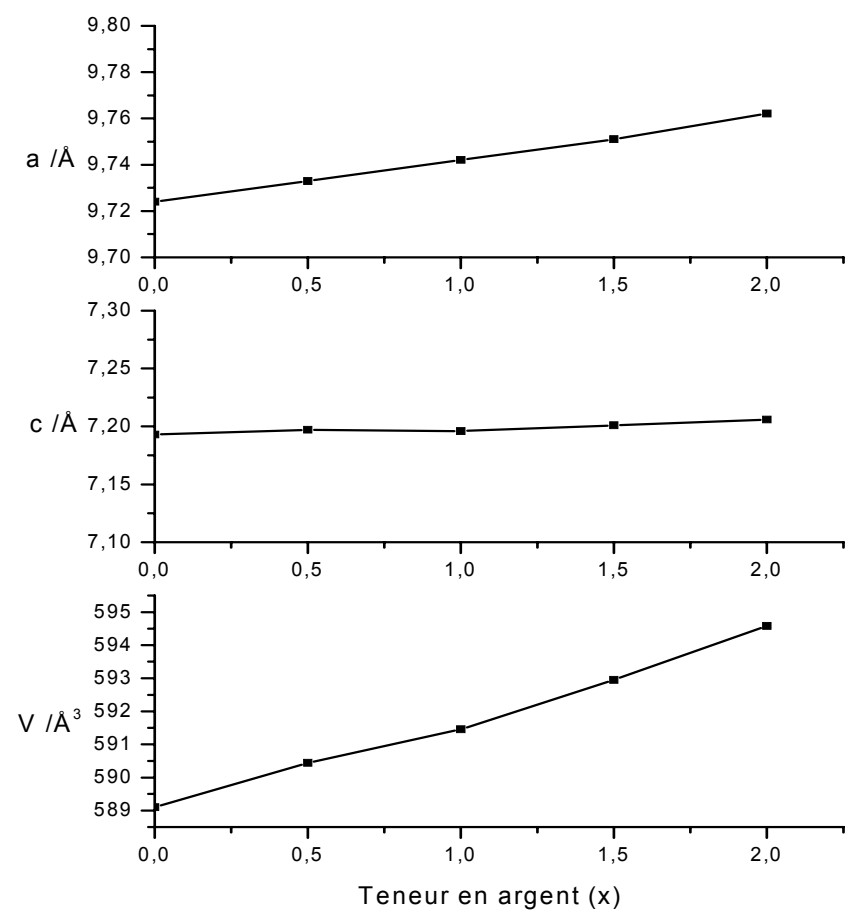

Figure 1. Variation des paramètres a, $c$ et du volume $\mathrm{V}$ de la maille des apatites $\mathrm{Pb}_{8} \mathrm{Na}_{2-\mathrm{x}} \mathrm{Ag}_{\mathrm{x}}\left(\mathrm{PO}_{4}\right)_{6}(0 \leq \mathrm{x} \leq 2)$.

Les spectres d'absorption IR et de diffusion Raman des échantillons présentent des bandes attribuées aux vibrations des groupements $\mathrm{PO}_{4}^{3-}$ dans une structure apatitique. L'absence des groupements hydroxyle $\mathrm{OH}^{-}$susceptibles de former des hydroxyapatites est mise en évidence par l'absence de la bande de libration $v_{\mathrm{L}}(\mathrm{OH})$ vers $630 \mathrm{~cm}^{-1}$ dans le spectre d'absorption IR et des bandes de vibration de valence $v_{\mathrm{S}}(\mathrm{OH})$ vers $3570 \mathrm{~cm}^{-1}$ dans les spectres d'absorption IR et de diffusion Raman.

Il a été mis en évidence un déplacement vers les faibles nombres d'onde des bandes relatives aux modes de vibration des ions $\mathrm{PO}_{4}^{3-}$ quand la teneur en argent augmente ; ceci est lié à l'expansion du volume de maille et à la variation de la nature des interactions entre le métal ( $\mathrm{Na}$ et $\mathrm{Ag}$ ) et l'oxygène. Les valeurs des déplacements chimiques extraites des spectres $\mathrm{RMN}$ du ${ }^{31} \mathrm{P}$ réalisés en MAS à $8 \mathrm{kHz}$ et en utilisant le carbonate de sodium $\mathrm{Na}_{2} \mathrm{CO}_{3}$ comme diluant des apatites phosphoplombeuses mixtes d'argent et de sodium, sont reportées dans le tableau 1.

Les spectres présentent diverses raies révélant l'existence, dans ces composés, de plusieurs environnements du phosphore. Tous les spectres sont dominés par une composante majoritaire (notée raie 1 dans le tableau 1) centrée autour 2-2,5 ppm. Elle peut être identifiée avec l'environnement du phosphore dans l'apatite pure, comme il a été mentionné pour l'hydroxyapatite de calcium $\left(\delta_{\text {iso }}=2,87\right.$ ppm) [8]. Les autres raies de déplacement chimique négatif sont attribuées à des phosphores non équivalents.

La présence de plusieurs environnements du ${ }^{31} \mathrm{P}$ dans ces composés traduit une distorsion des tétraèdres $\mathrm{PO}_{4}$, probablement due à la localisation des ions $\mathrm{Na}^{+}$et $\mathrm{Ag}^{+}$en site (I) et à la présence de lacunes anioniques [9]. 
Tableau 1. Déplacements chimiques des spectres RMN-MAS du ${ }^{31} \mathrm{P}$ des apatites $\mathrm{Pb}_{8} \mathrm{Na}_{2-\mathrm{x}} \mathrm{Ag}_{\mathrm{x}}\left(\mathrm{PO}_{4}\right)_{6}(0 \leq \mathrm{x} \leq 2)$.

\begin{tabular}{|l|c|c|}
\hline \multicolumn{1}{|c|}{ Echantillon } & Raie & $\delta_{\text {iso }} / \mathrm{ppm}$ \\
\hline \multirow{3}{*}{$\mathrm{E}_{1}(\mathrm{x}=0)$} & 1 & 2,068 \\
& 2 & $-1,016$ \\
& 3 & $-8,578$ \\
& 4 & $-11,380$ \\
$\mathrm{E}_{2}(\mathrm{x}=0,5)$ & 5 & $-11,880$ \\
\hline $\mathrm{E}_{3}(\mathrm{x}=1)$ & 1 & 2,136 \\
& 3 & $-7,229$ \\
\hline $\mathrm{E}_{4}(\mathrm{x}=1,5)$ & 1 & 2,228 \\
& 3 & $-7,955$ \\
\hline \multirow{3}{*}{$\mathrm{E}_{5}(\mathrm{x}=2)$} & 1 & 2,262 \\
& 3 & $-8,608$ \\
\hline
\end{tabular}

\subsection{Propriétés électriques}

Les diagrammes d'impédance complexe des apatites mixtes d'argent et de sodium $\mathrm{Pb}_{8} \mathrm{Na}_{2-\mathrm{x}} \mathrm{Ag}_{\mathrm{x}}\left(\mathrm{PO}_{4}\right)_{6}$ $(0 \leq \mathrm{x} \leq 2)$, dans la gamme de fréquences $5 \mathrm{~Hz}-13 \mathrm{MHz}$, sont représentés par des demi-cercles centrés sur l'axe représentant les parties réelles de l'impédance (Figure 2). A faible teneur en argent $(\mathrm{x}=0-0,5)$, les diagrammes d'impédance complexe sont formés de deux arcs de cercle. Le premier arc est relatif à la conduction en volume (intérieur des grains) alors que le deuxième représente la conduction par les joints de grains [10]. Dans ce travail, nous nous limitons à l'étude de la conductivité ionique en volume en prenant l'intersection avec l'axe des réels de la continuité du premier arc de cercle. Au fur et à mesure que la teneur en argent $(x)$ augmente et pour des températures élevées, la courbe associée à la fonction $\left(-Z^{\prime \prime}=\mathrm{f}\left(Z^{\prime}\right)\right)$ n'est plus circulaire et présente une allure caractéristique d'une conduction électronique.

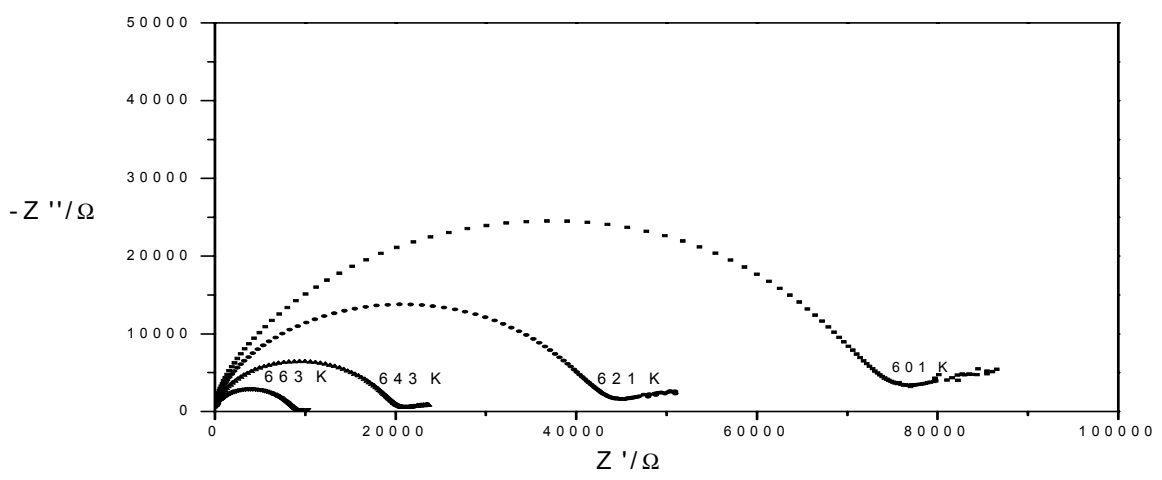

Figure 2. Evolution des diagrammes d'impédance complexe en fonction de la température de $\mathrm{Pb}_{8} \mathrm{AgNa}\left(\mathrm{PO}_{4}\right)_{6}$. 
Les diagrammes d'Arrhenius qui représentent les variations du Ln $(\sigma \mathrm{T})$ en fonction de l'inverse de la température sont donnés dans la figure 3. Il est à remarquer que, dans le domaine étudié des températures (473-804 K), la conductivité des apatites $\mathrm{Pb}_{8} \mathrm{Na}_{2-\mathrm{x}} \mathrm{Ag}_{\mathrm{x}}\left(\mathrm{PO}_{4}\right)_{6}(0 \leq \mathrm{x} \leq 2)$ vérifie la loi d'Arrhenius. Les valeurs moyennes des énergies d'activation extraites des diagrammes d'Arrhenius sont données dans le tableau 2.

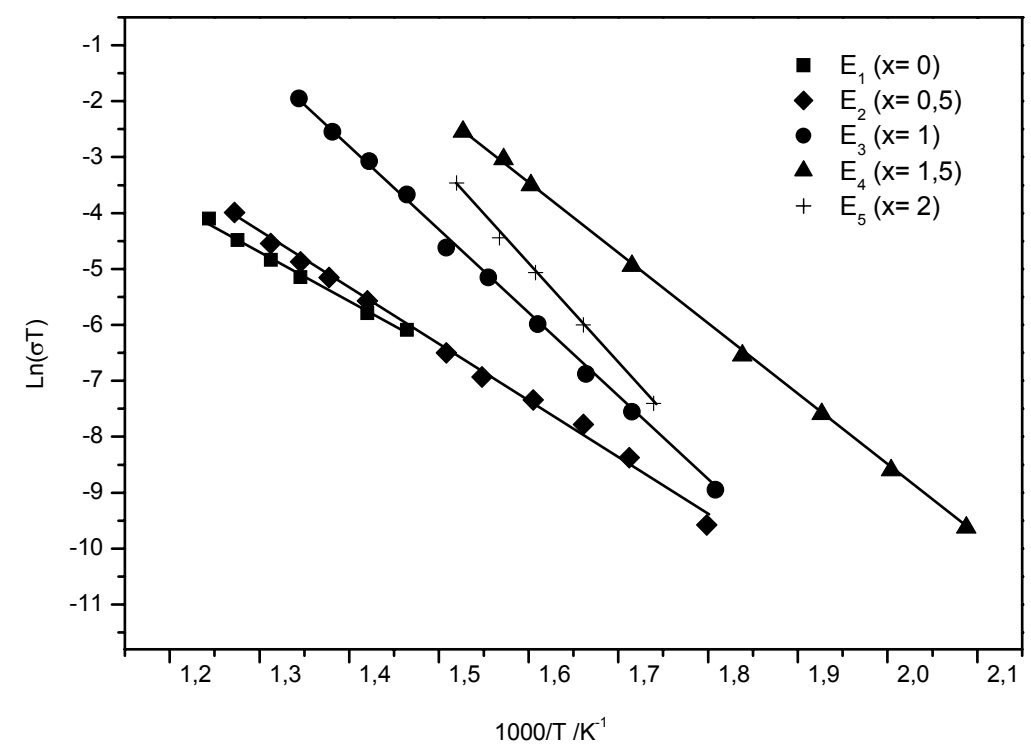

Figure 3. Diagrammes d'Arrhenius des apatites $\mathrm{Pb}_{8} \mathrm{Na}_{2-\mathrm{x}} \mathrm{Ag}_{\mathrm{x}}\left(\mathrm{PO}_{4}\right)_{6}(0 \leq \mathrm{x} \leq 2)$.

Tableau 2. Energies d'activation des apatites $\mathrm{Pb}_{8} \mathrm{Na}_{2-\mathrm{x}} \mathrm{Ag}_{\mathrm{x}}\left(\mathrm{PO}_{4}\right)_{6}(0 \leq \mathrm{x} \leq 2)$.

\begin{tabular}{|l|l|}
\hline Echantillon & $E_{a}(e V)$ \\
\hline$E_{1}(x=0)$ & 0,77 \\
\hline$E_{2}(x=0,5)$ & 0,87 \\
\hline$E_{3}(x=1)$ & 1,30 \\
\hline$E_{4}(x=1,5)$ & 1,10 \\
\hline$E_{5}(x=2)$ & 1,53 \\
\hline
\end{tabular}

Il est à noter que vers les hautes températures $(\mathrm{T} \geq 655 \mathrm{~K})$, la conductivité est une fonction croissante avec la teneur en argent jusqu'à $\mathrm{x}=1,5$ qui correspond à la composition du meilleur conducteur.

Dans ces matériaux, la conduction électrique est reliée à la mobilité des cations dans les tunnels de l'apatite, par analogie avec les travaux de Den Hartog qui a démontré, pour la fluoroapatite que la conductivité dominante est celle liée à la mobilité des cations [11]. En plus, dans des études récentes sur les apatites de plomb sans anions $\mathrm{Pb}_{8} \mathrm{~K}_{2}\left(\mathrm{PO}_{4}\right)_{6}$ [12] et $\mathrm{Pb}_{6} \mathrm{Ca}_{2} \mathrm{Li}_{2}\left(\mathrm{PO}_{4}\right)_{6}$ [13], les auteurs ont montré que seuls les cations dans les sites I sont responsables de la conductivité ionique. 
Le mécanisme de mobilité des cations monovalents $\left(\mathrm{Na}^{+}\right.$et $\left.\mathrm{Ag}^{+}\right)$situés dans les sites I peut être expliqué par la création de défauts qui sont thermiquement activés comme les défauts de Frenkel. Ces derniers sont des défauts ponctuels constitués d'un ensemble formé d'une lacune cationique et d'un cation interstitiel.

L'augmentation de la conductivité avec la teneur en argent, montre que le phénomène de conduction au sein de ces matériaux, est gouverné par un autre facteur autre que la taille des cations. En effet, l'introduction de l'ion $\mathrm{Ag}^{+}$plus volumineux que l'ion $\mathrm{Na}^{+}$, doit avoir pour conséquence la diminution de la conductivité or c'est le cas contraire qui est observé. Ce facteur pourrait être expliqué par la contribution de la conductivité électronique. En effet, à forte teneur en argent et pour des températures élevées, la courbe associée à la fonction $\left(-Z^{\prime \prime}=\mathrm{f}\left(\mathrm{Z}^{\prime}\right)\right)$ n'est plus circulaire. Nous pouvons conclure que dans ces matériaux et à mesure que la teneur en argent augmente, la conduction devient mixte : ionique et électronique.

Par rapport à d'autres apatites de plomb, il est à conclure que les apatites mixtes d'argent et de sodium $\mathrm{Pb}_{8} \mathrm{Na}_{2-\mathrm{x}} \mathrm{Ag}_{\mathrm{x}}\left(\mathrm{PO}_{4}\right)_{6}(0 \leq \mathrm{x} \leq 2)$ sont des conducteurs de performances électriques comparables.

\section{CONCLUSION}

Une série de solution solide continue d'apatites de plomb mixtes d'argent et de sodium de formule générale $\mathrm{Pb}_{8} \mathrm{Na}_{2-\mathrm{x}} \mathrm{Ag}_{\mathrm{x}}\left(\mathrm{PO}_{4}\right)_{6}(0 \leq \mathrm{x} \leq 2)$ a été synthétisée par réaction à l'état solide. L'analyse chimique, la diffraction des rayons $\mathrm{X}$ et les spectroscopies d'absorption infrarouge et de diffusion Raman ont été utilisées pour la caractérisation complète des matériaux. La résonance magnétique nucléaire à l'état solide montre la présence de plusieurs environnements du phosphore traduisant une distorsion des tétraèdres $\mathrm{PO}_{4}$. Une étude de la conductivité ionique en se basant sur la technique d'impédance complexe dans l'intervalle de températures 473-804 K a été entreprise. Il a été mis en évidence une augmentation de la conductivité ionique avec la teneur en argent. Au sein de ces matériaux, à mesure que la teneur en argent augmente, la conduction devient mixte : ionique et électronique.

\section{Références}

[1] St. Naray-Szabo, Zeitsch-Krist., 75 (1930) 387.

[2] M. Quarton, M.T. Oumba, W. Freundlich and A.W. Kolsi, Mat. Res. Bull., 19 (1984) 1063.

[3] I. Mayer and S. Swissa, J. Less-Common Metals, 110 (1985) 411.

[4] R. Ternane, M. Ferid, M. Trabelsi-Ayadi and B. Piriou, Spectrochim. Acta, A(55) (1999) 1793.

[5] R. Ternane, M. Ferid, N. Kbir-Ariguib and M. Trabelsi-Ayadi, J. All. Comp., 308 (2000) 83.

[6] A. Laghzizil, N. Herch, A. Bouhaouss, G. Lorente and J. Macquette, J. Solid State Chem., 156 (2001) 57.

[7] A. Gee and V.R. Deitz, Anal. Chem., 25 (1953) 1320.

[8] W.P. Rothwell, J.S. Waugh et J.P. Yesinowski, J. Am. Chem. Soc., 102 (1980) 2637.

[9] P.R. Suitch, J.L. Lacout, A. Hewat et RA. Young, Acta Cryst. B, 41 (1985) 173.

[10] J.R. Macdonald, "Impedance spectroscopy, Emphasizing Solid Materials and Systems", Wiley Interscience, New York (1987).

[11] H. Den Hartog, Phys. Stat. So. (b) 53 (1972) 201.

[12] A. Laghzizil, P. Barboux and A Bouhaouss, Solid State Ionics, 128 (2000) 177.

[13] Th. Naddari, J.M. Savariault, H. El Feki, P. Salles and A. Ben Salah, J. Solid State Chem., 166 (2002) 237. 